\title{
Two Novel Methods for Parameter Identification of Synchronous Machine Using DC-Decay Test with Rotor in Arbitrary Position
}

\author{
Frederic Maurer, Mai Tu Xuan and Jean-Jacques Simond, Member, IEEE
}

\begin{abstract}
The goal of this paper is to propose and validate standstill DC-Decay measuring methods for a rotor in arbitrary position. The first new method (DC-Decay I) delivers the equivalent circuit with a few measurements. The second new method (DC-Decay III) permits to catch the full equivalent circuit of both axes in only one measurement which is unique and a breakthrough in parameter identification. A new method (DC-Decay II) is used to get the rotor angle. The field current allows to obtain the characteristic reactance $x_{c}$. To extract the time constants and the reactances an enhancement of "classical" parameter identification algorithms is presented (used for the DC-Decay I method). Simulation and experimental results validate the methods and show their accuracy and drawback.
\end{abstract}

Index Terms-Electric variables measurement, Equivalent circuits, Iterative algorithms, Generators, Power system dynamics.

\section{NOMENCLATURE}

In this paper, the following nomenclature is used:

- $r_{s}, r_{f}, r_{D}, r_{Q}$ armature, field winding, d-axis and q-axis damper winding resistance, p.u.;

- $x_{d}, x_{q}, x_{d}^{\prime}, x_{d}^{\prime \prime}, x_{q}^{\prime \prime}$ synchronous, transient and subtransient reactance of the d-axis and q-axis, p.u.;

- $x_{a d}, x_{a q}$ main reactances, p.u.;

- $x_{c}$ characteristic reactance [1], p.u.;

- $T_{d}^{\prime}, T_{d}^{\prime \prime}, T_{q}^{\prime \prime}$ transient and subtransient short-circuit time constants, seconds;

- $T_{d 0}^{\prime}, T_{d 0}^{\prime \prime}, T_{q 0}^{\prime \prime}$ transient and subtransient open-circuit time constants, seconds;

- $T_{\sigma D}, T_{\sigma f}$ leakage time constant of the d-axis damper, field winding, seconds;

- $p=\frac{d}{d t}, \omega_{n}=2 \pi f_{n}$, Laplace operator, Rated pulsation;

- $\theta$, Angle between the d-axis and the phase of stator phase a (rotor angle), rad.

[2] gives the definition of the used equivalent circuits with only one damper circuit per axis, used in this paper.

\section{INTRODUCTION}

The analysis of transient behaviour of synchronous machines requires a precise knowledge of the characteristic quantities of the machine, especially the subtransient reactances. There are many methods to obtain these quantities through normalized procedures [3]. While DC-Decay tests

The authors are with Ecole Polytechnique Federale de Lausanne, Switzerland (e-mail: frederic.maurer@epfl.ch). represent a non-normalized alternative [4]. This tests require less time, less power and a much smaller short-circuit breaker than other methods. The main handicap of DC-Decay tests lies in the necessity to align the rotor with the $\mathrm{d}$ or $\mathrm{q}$ axis which is difficult for big units. Current publications emanate from the hypothesis that the rotor is aligned with the $\mathrm{d}$ or $\mathrm{q}$ axis, or present a procedure to align the rotor with the main axes [5]- [13]. Reference [14] presents a method to obtain the characteristic quantities with the rotor in an arbitrary position. The modelling of the short-circuit was not precise enough so that some discrepancies appeared between the identified parameters and the theoretical ones.

\section{THEORY - TRANSFER FUNCTIONS}

\section{A. DC-Decay I}

Starting from the voltage equations for $u_{a}$ and $u_{b}$ presented in [15] with $i_{c}=0$ and $i_{b}=-i_{a}$ one can obtain the transfer function for the test circuit presented in Fig. 1.

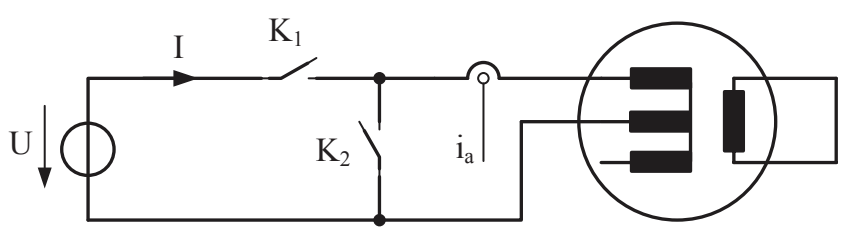

Fig. 1: Block diagram for the DC-Decay I method

Section VI describes the operation of the switches $K_{1}$ and $K_{2}$. The transfer function is valid for a random rotor position and is calculated assuming a short-circuit between the stator phases A and B. Considering a laminated salient pole synchronous generator, the transfer function for small variations is given by

$$
i_{a}(p)=\frac{R_{f}(p) R_{Q}(p)}{R_{a b}(p)} u_{a b}(p) .
$$

$R_{a b}(p)$ is defined by

$$
\begin{aligned}
R_{a b}(p)= & 2\left[r_{s}+\frac{1}{3} p\left(\alpha^{2} \frac{x_{d}}{\omega_{n}}+\beta^{2} \frac{x_{q}}{\omega_{n}}\right)\right] R_{f}(p) R_{Q}(p) \\
& -R_{Q}(p) \frac{2}{3} \frac{\alpha^{2} x_{a d}^{2}}{\omega_{n}^{2}}\left(\frac{1+p T_{\sigma D}}{r_{f}}+\frac{1+p T_{\sigma f}}{r_{D}}\right) p^{2} \\
& -R_{f}(p) \frac{2}{3} \frac{\beta^{2} x_{a q}^{2}}{r_{Q} \omega_{n}^{2}} p^{2}
\end{aligned}
$$


where $\alpha=\cos (\theta)-\cos \left(\theta-\frac{2 \pi}{3}\right)$ and $\beta=\sin (\theta)-\sin \left(\theta-\frac{2 \pi}{3}\right)$. The polynomal $R_{f}(p)$ and $R_{Q}(p)$ are given by

$$
\begin{aligned}
& R_{f}(p)=\left(1+p T_{d 0}^{\prime}\right)\left(1+p T_{d 0}^{\prime \prime}\right), \quad \text { and } \\
& R_{Q}(p)=1+p T_{q 0}^{\prime \prime} .
\end{aligned}
$$

The coupling between the two axes is non-linear and takes place in the equation of $R_{a b}(p)$ through the constants $\alpha$ and $\beta$. To obtain the equivalent circuit for both axes, one must consider the angular variation ("root-locus") of the four zeros of $R_{a b}(p)$. This root-locus of the four zeros exhibits four minima and four maxima, which correspond to a zero or pole of the transfer functions in the d- or q-axis [16]. There will be some pole/zero simplifications, as the transfer function for a machine aligned with one axis, has less poles and zeros than the transfer function represented in equation (1). This fact complicates the estimation of the maxima and minima of the root-locus.

\section{B. DC-Decay II}

Fig. 2 shows the test circuit used for the DC-Decay II method.

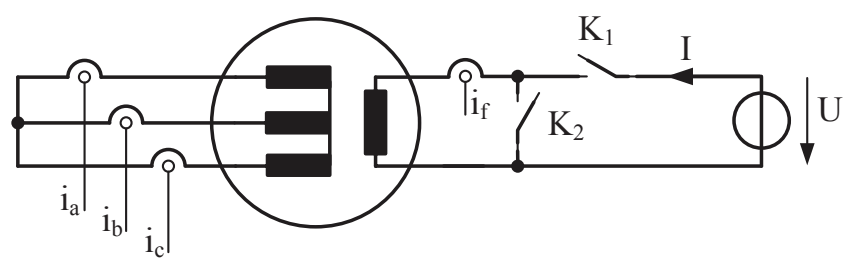

Fig. 2: Block diagram for the DC-Decay II method

This test circuit is used to obtain the rotor position by measuring the armature current decay. With this test circuit one could obtain the parameters of the equivalent circuit in the d-axis. For a perfect symmetrical machine, only the d-axis is excited during this test. Therefore assuming that the q-axis component of the armature current is zero, one can compute the rotor position $(\theta)$ by solving the following equation

$$
\begin{aligned}
0=i_{q}= & -\frac{2}{3}\left(i_{a} \sin (\theta)+i_{b} \sin \left(\theta-\frac{2 \pi}{3}\right)+\ldots\right. \\
& \left.+i_{c} \sin \left(\theta+\frac{2 \pi}{3}\right)\right)
\end{aligned}
$$

after some simplification one obtains

$$
\tan (\theta)=\frac{\sqrt{3}\left(i_{b}-i_{c}\right)}{2 i_{a}-i_{b}-i_{c}}
$$

\section{DC-Decay III}

To obtain a more practical solution, one need to find a simpler way to decouple the current response for both axes than the one used in the DC-Decay I method. At standstill, the transient expressions for a synchronous machine are given by

$$
u_{d}=r_{s} i_{d}+\frac{p}{\omega_{n}} x_{d} \frac{\left(1+p T_{d}^{\prime}\right)\left(1+p T_{d}^{\prime \prime}\right)}{\left(1+p T_{d 0}^{\prime}\right)\left(1+p T_{d 0}^{\prime \prime}\right)} i_{d}
$$

$$
u_{q}=r_{s} i_{q}+\frac{p}{\omega_{n}} x_{q} \frac{1+p T_{q}^{\prime \prime}}{1+p T_{q 0}^{\prime \prime}} i_{q}
$$

Because the coupling is due to either non-equal impedances in each phases of the stator or to the rotation induced voltage $\left(\omega \Psi_{q}\right.$ and $\omega \Psi_{d}$ ), there is no coupling between both axes. A three-phase short-circuit yields to a simultaneous decaying in both axes. Suffisant current level for both axes guarantees homogenous characteristic quantities. Finally the DC-Decay II method gives the rotor angle. Fig. 3 shows the test circuit used for the DC-Decay III method, which is also used in [5].

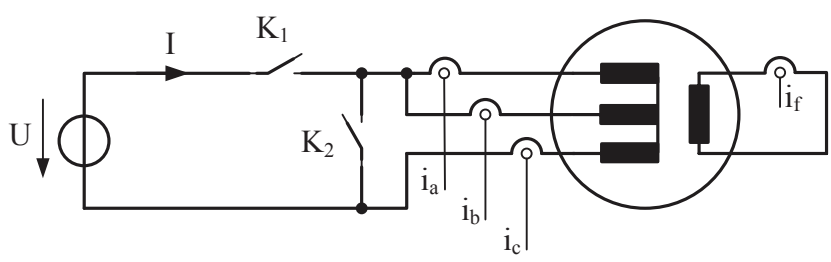

Fig. 3: Block diagram for the DC-Decay III method

Solving equation (7) for $i_{d}$ leads to

$$
i_{d}(p)=\frac{\left(1+p T_{d 0}^{\prime}\right)\left(1+p T_{d 0}^{\prime \prime}\right)}{R_{d}(p)} \frac{u_{d}(p)}{r_{s}}
$$

where $R_{d}(p)$ is given by

$$
R_{d}(p)=R_{f}(p)+p T_{d}\left(1+p T_{d}^{\prime}\right)\left(1+p T_{d}^{\prime \prime}\right)
$$

and $T_{d}$ is defined by $\frac{x_{d}}{\omega_{n} r_{s}}$. Proceeding the same way for the $\mathrm{q}$-axis leads to the following expression for $i_{q}(p)$

$$
i_{q}(p)=\frac{\left(1+p T_{q 0}^{\prime \prime}\right)}{\left(1+p T_{q 0}^{\prime \prime}\right)+p T_{q}\left(1+p T_{q}^{\prime \prime}\right)} \frac{u_{q}(p)}{r_{s}}
$$

where $T_{q}=\frac{x_{q}}{\omega_{n} r_{s}}$. These transfer functions can be easily adapted for different expressions of the reactance operators ( [1]) or number of rotor circuits for a particular axis.

\section{Determination of $x_{c}$ - characteristic reactance}

The determination of $x_{c}$ is done using a similar procedure as presented in [1]. The transfer function between the d-axis armature current and the field current is given by

$$
i_{f}(p)=-\frac{p x_{a d}\left(1+p T_{\sigma D}\right)}{r_{f} \omega_{n}\left(1+T_{d 0}^{\prime}\right)\left(1+T_{d 0}^{\prime \prime}\right)} i_{d}(p) .
$$

The time constant $T_{\sigma D}$ is identified using the field current. Assuming a certain value for $x_{c}$ the equivalent circuit is computed using the identified characteristic quantities. $T_{\sigma D}$ is computed from the equivalent circuit. If the error between the identified $T_{\sigma D}$ and the computed one is less than a given value, then the assumed value of $x_{c}$ is correct. If the error is too high, then the assumed value of $x_{c}$ is increased by a given step and the procedure is repeated up to convergence. 


\section{PARAMETER IDENTIFICATION ALGORITHM}

For the method DC-Decay II, there is not special identification algorithm, equation (6) is applied to get the rotor position. For the DC-Decay III method, the zeros and poles of the transfer function are obtained fitting a curve (see also [7] or [11]) with two or three time constants (see equation (9) and (11)) on the time-dependant stator current curves projected in Park's reference frame.

\section{A. DC-Decay I}

The identification is done according to different steps shown in Fig. 4.

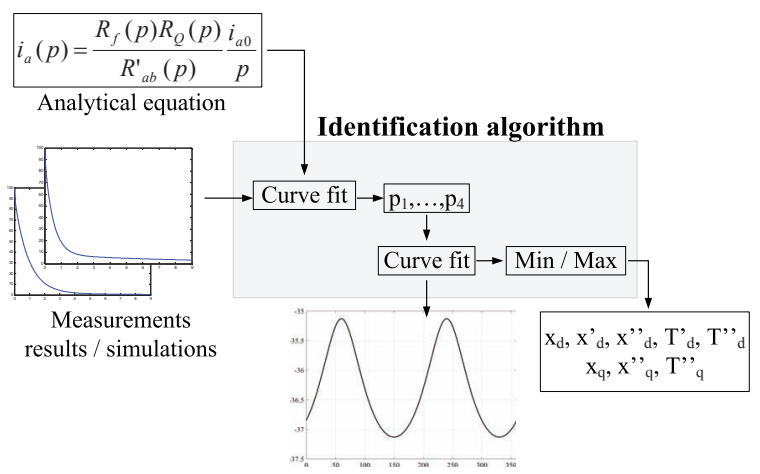

Fig. 4: Structural diagram of the parameter identification algorithm

The measured currents are fitted with the analytical expression of the transfer function to get the poles in a similar way as presented in [7] or [11]. The poles are plotted into the root-locus. This procedure is repeated for each measurement. A second curve fit with Fourier series ${ }^{1}$ based on the rootlocus allows to obtain the maxima and minima of the rootlocus from which, the characteristic quantities. This leads to a rather sophisticated and noise sensitive method, as it requires a certain number of "perfect" measurements to achieve low error. The pole/zero simplifications in equation (1) lead to huge identification errors of the pole when the rotor is aligned with one axis as in Fig. 5 (pole $\beta$ ). Every pole in not affected the same way.

To eliminate them, one uses an advanced technique to get a more precise approximation of the maxima and minima in the root locus. The structural diagram of the enhanced algorithm is depicted in Fig. 6. The four poles are within different time scales for large generators $(0.02 \mathrm{~s}, 0.05 \mathrm{~s}, 0.5 \mathrm{~s}$, $10 \mathrm{~s})$ so that the current curves can be splited into intervals where only the dominant pole over this interval is identified. In addition, the time interval for the "single"-pole identification is corrected if the SSE (Sum of Squared Error) is higher than a given threshold. The time interval correction act as a temporal filter, which helps to focus on the pole to be identified. This lead to a very robust identification algorithm.

${ }^{1}$ Using Ferrari's method, it is possible to demonstrate, that the roots of the polynom $R_{a b}(p)$ can be expressed with a Fourier series.
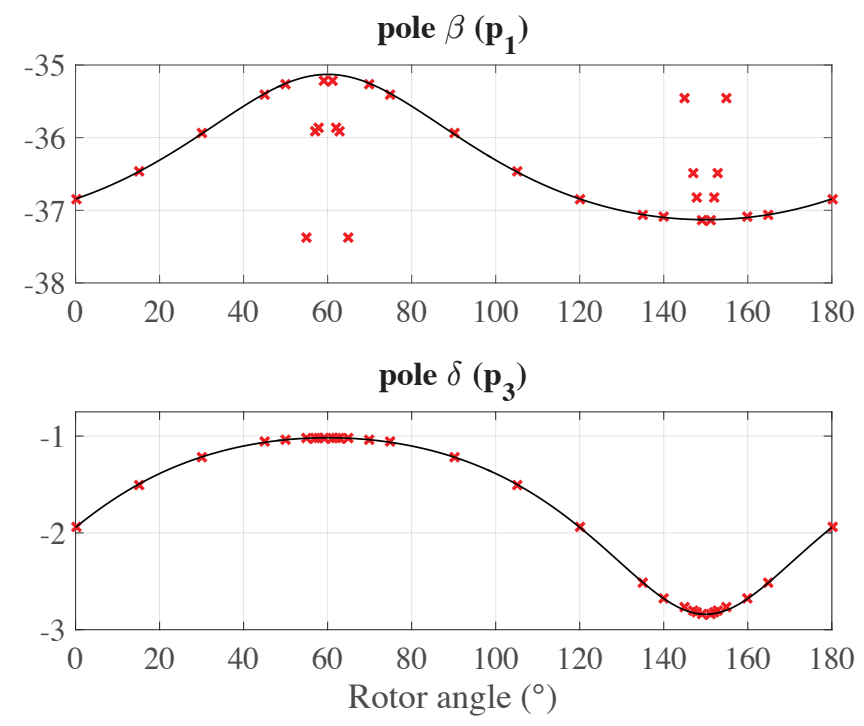

Fig. 5: Root locus for 2 zeros of $R_{a b}(p)$. Black curve: calculated poles, red crosses: identified poles of SM1 (simulation without noise, $180 \mathrm{MVA}, 13.8 \mathrm{kV}, 150 \mathrm{rpm}, 50 \mathrm{~Hz})$. Q-axis at $60^{\circ}$.

\section{Identification algorithm}

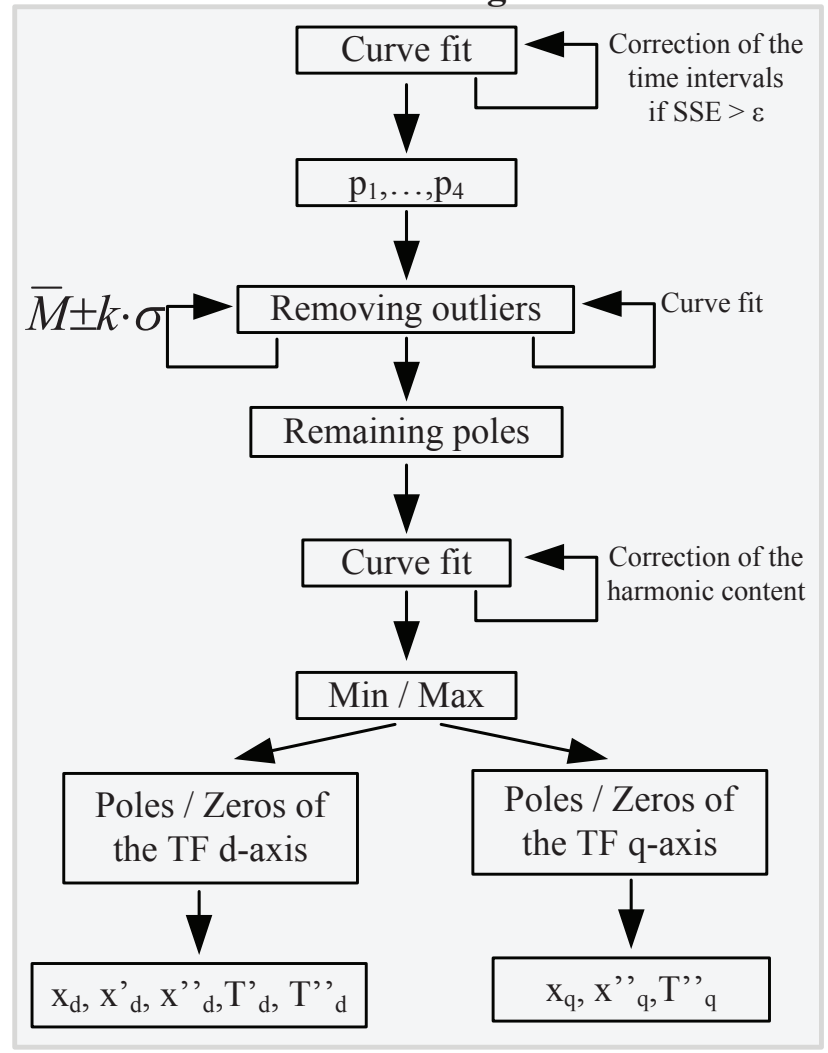

Fig. 6: Details of the identification algorithm. 
The exist also outliers as depicted in Fig. 5, which extraction is crucial for the precision of the parameter idenficiation. To extract them, one use a binary selection based on a mean-value $(\bar{M}) \pm \sigma$ (standard deviation) criterion as the pole behaves like a sinus-curve over the angle. A second filtration is based on a Fourier-space interpolation (with low harmonic content) and selection of the outliers using a criteria of distance between the identified pole and its interpolated value. The harmonic content can be adapted to enhance the filtration of the outliers.

\section{Simulation RESUlts}

The simulation results are obtained assuming perfect switches. $K_{1}$ and $K_{2}$ are always switched at the same time removing any problem arising from the commutation. The used simulations models are linear. All simulations have been performed without any noise (gaussian noise, white noise). The voltage source is an element with a constant voltage for any time. The simulations are done using SIMSEN or MATLAB, while we used a space-state model for the MATLAB simulations.

\section{A. DC-Decay I}

We carry out many simulations varying the sampling period from $0.2 \mathrm{~ms}$ to $1 \mathrm{~ms}$, the harmonic content of the root locus interpolation function from 2 to 12 harmonics and the angular step from $1^{\circ}$ to $20^{\circ}$. Table I presents the minimum and the maximum error obtained for all simulations.

TABLE I: DC-Decay I: Parameter identification results minimal and maximal error for the machine SM1.

\begin{tabular}{lll}
\hline Parameter & $\begin{array}{l}\text { Identification }^{a} \\
(\text { Error [\%]) }\end{array}$ & $\begin{array}{l}\text { Identification }^{b} \\
\text { (Error [\%]) }^{b}\end{array}$ \\
\hline$x_{d}$ [p.u.] & $1.0255(0.0458)$ & $1.0275(0.2436)$ \\
$x_{d}^{\prime}$ [p.u.] & $\mathbf{0 . 2 9 5 4}(\mathbf{0 . 2 1 2 0})^{c}$ & $0.2975(0.5112)$ \\
$x_{d}^{\prime \prime}$ [p.u.] & $0.1983(0.1669)$ & $0.1993(0.6496)$ \\
$T_{d}^{\prime}$ [s] & $2.5663(0.1276)$ & $2.5798(0.6553)$ \\
$T_{d}^{\prime \prime}$ [s] & $0.0280(0.0066)$ & $0.0280(0.0084)$ \\
$x_{q}$ [p.u.] & $0.7087(0.0492)$ & $\mathbf{0 . 7 1 5 1 ( 0 . 8 5 5 9 )}$ \\
$x_{q}^{\prime \prime}$ [p.u.] & $0.2019(0.0404)$ & $0.2035(0.7616)$ \\
$T_{q}^{\prime \prime}$ [s] & $0.0310(0.0114)$ & $0.0310(0.0931)$ \\
$x_{c}$ [p.u.] & $0.1148(0.1739)$ & $0.1151(0.0870)$ \\
\hline
\end{tabular}

${ }^{a}$ Sampling period of $0.2 \mathrm{~ms}$ and angular step of $10^{\circ}$

${ }^{b}$ Sampling period of $1.0 \mathrm{~ms}$ and angular step of $25^{\circ}$.

${ }^{c} x_{d}^{\prime}$ is calculated using the simplified expression $x_{d}^{\prime} \cong \frac{x_{d} T_{d}^{\prime}}{T_{d 0}^{\prime}}$.

The maximum value of the error is below $1 \%$ showing a very precise method, even if there is some issues regarding pole/zero cancellation. The sampling period influence the angular width of the zone where the outliers are generated, but every pole is affected differently. An increase of the sampling frequency from $1 \mathrm{kHz}$ to $5 \mathrm{kHz}$ can lead to a reduction of the angular width up to $10^{\circ}$ for SM1. Using at least 8 harmonics in the Fourier-series reduces the identification error to less than $1 \%$ for any angular step. The optimal angular step was found to be situated between $5^{\circ}$ and $10^{\circ}$ and doesn't need to be equally distributed.

\section{B. DC-Decay II}

We accomplish simulations at four different angles, namely $16.062^{\circ}, 146.9^{\circ}, 260.82^{\circ}$ and $333.333^{\circ}$ changing the number of samples used for the mean value calculation of the angle from 10 up to $10^{\prime} 000$. For a perfectly symmetric machine (SM1), the maximal value of the error is $11 \mathrm{ppm}$. The number of samples does not impact the error. If the stator phases are not symmetric, a non-zero q-axis current remains. To highlight the impact of this non-symmetry on the angle calculation, a resistance was introduced in one stator phase. For a dis-symmetry of $10 \%$ (adding a resistance of $10 \%$ of one phase resistance) the error increases to 543ppm. Even with a high dis-symmetry, this method has a negligible error.

\section{DC-Decay III}

The simulation results are shown in table II.

TABLE II: DC-Decay III: Parameter identification results minimal and maximal error for the machine SM1.

\begin{tabular}{lll}
\hline Parameters & $\theta=16.062^{\circ}$ & $\theta=146.9^{\circ}$ \\
\hline$x_{d}$ [p.u.] & $1.0253(0.0323)^{a}$ & $1.0253(0.0317)$ \\
$x_{d}^{\prime}$ [p.u.] & $\mathbf{0 . 2 9 4 9}^{b}(\mathbf{0 . 3 6 7 2})$ & $\mathbf{0 . 2 9 4 9}(\mathbf{0 . 3 6 8 4})$ \\
$x_{d}^{\prime \prime}$ [p.u.] & $0.1982(0.1238)$ & $0.1982(0.0875)$ \\
$T_{d}^{\prime}$ [s] & $2.5626(0.0155)$ & $2.5625(0.0176)$ \\
$T_{d}^{\prime \prime}$ [s] & $0.0280(0.0008)$ & $0.0280(0.0953)$ \\
$x_{q}$ [p.u.] & $0.7092(0.0277)$ & $0.7092(0.0289)$ \\
$x_{q}^{\prime \prime}$ [p.u.] & $0.2025(0.2274)$ & $0.2026(0.3031)$ \\
$T_{q}^{\prime \prime}$ [s] & $0.0310(0.0337)$ & $0.0310(0.0725)$ \\
\hline
\end{tabular}

${ }^{a}$ Identification error in $\%$.

${ }^{b} x_{d}^{\prime}$ is calculated using the approximative relation $x_{d}^{\prime} \cong \frac{x_{d} T_{d}^{\prime}}{T_{d 0}^{\prime}}$.

The theoretical identification error is about $0.37 \%$. Some simulations have been performed to illustrate the influence of a current non-symmetry. The dys-symmetry was modelled adding a resistance in one of the branches in parallel of the stator winding. The identification error raises to $19.85 \%$ for $T_{d}^{\prime \prime}$ and to $5.70 \%$ for $x_{d}^{\prime \prime}$ for a current non-symmetry of $2.5 \%$ (the current in one branch is $2.5 \%$ higher than the equilibrium current). The current dys-symmetry influences the parameter identification in a significant way.

\section{EXPERIMENTAL METHOD}

\section{A. Operation of the switches $K_{1}$ and $K_{2}$}

A $12 \mathrm{~V}$-battery with good short-circuit behaviour is the voltage source. A freewheeling diode in the experimental setup ensures a current path in any case. The measurements are performed respecting the subsequent process:

- Electrical connection according to the wanted block diagram;

- Closing switch $K_{1}$ to reach a stabilised current;

- Closing switch $K_{2}$ and opening switch $K_{1}$ (at the same time);

- Recording the current decay.

We use digital switches controlled by LabView to ensure an "as synchronous commutation as possible" of the switches, 
but we always close $K_{2}$ a little bit before opening $K_{1}$ to ensure a good catch of the subtransient current decay, as the freewheel diode is a poor electrical conductor.

\section{B. DC-Decay I}

To get the equivalent circuit for both axes, one needs to perform between 10 and 20 time the measurement process with a rotor displacement of $180^{\circ}$ between the first and the last measurement. The parameter identification algorithm (see section IV) gives the equivalent circuit for both axes.

\section{DC-Decay II}

Only one measurement is needed to acquire the rotor position.

\section{DC-Decay III}

The measurement steps are the following:

1) Determination of the rotor angular position (DC-Decay II method);

2) Choosing the optimal stator winding coupling (using Fig. 7);

3) Measurement;

4) Calculation of the d- and q-axis currents using Park's transformation;

5) Identification of the characteristic quantities from the curve-fitting of the currents $i_{d}$ and $i_{q}$.

The choice of the optimum stator winding coupling confers a minimal current above 1p.u. for every rotor angle in both axes. This insures a similar saturation level for both axes and therefore comparable reactances and time constants. There are three possible couplings of the stator winding which are presented in table III.

TABLE III: Different couplings of the stator winding.

\begin{tabular}{llll}
\hline & Phase a & Phase b & Phase c \\
\hline Coupling I & + & + & - \\
Coupling II & + & - & + \\
Coupling III & - & + & + \\
\hline
\end{tabular}

For example, the block diagram of Fig. 3 is coupled using Coupling I. Fig. 7 shows the amplitude of the initial $d$ and $\mathrm{q}$ axis currents in function of the rotor position for a current of 1 p.u.. Choosing an appropriate coupling for each angular position leads to the wanted minimal current for both axes. For example: with a measured angle of $120^{\circ}$ the optimal choice is coupling I and for an angle of $225^{\circ}$ the optimal choice is coupling III.

\section{EXPERIMENTAL RESULTS}

The measurements are done on the massive salient pole motor SM2 (200 kVA, 380 V, 1500 rpm, 50 Hz).

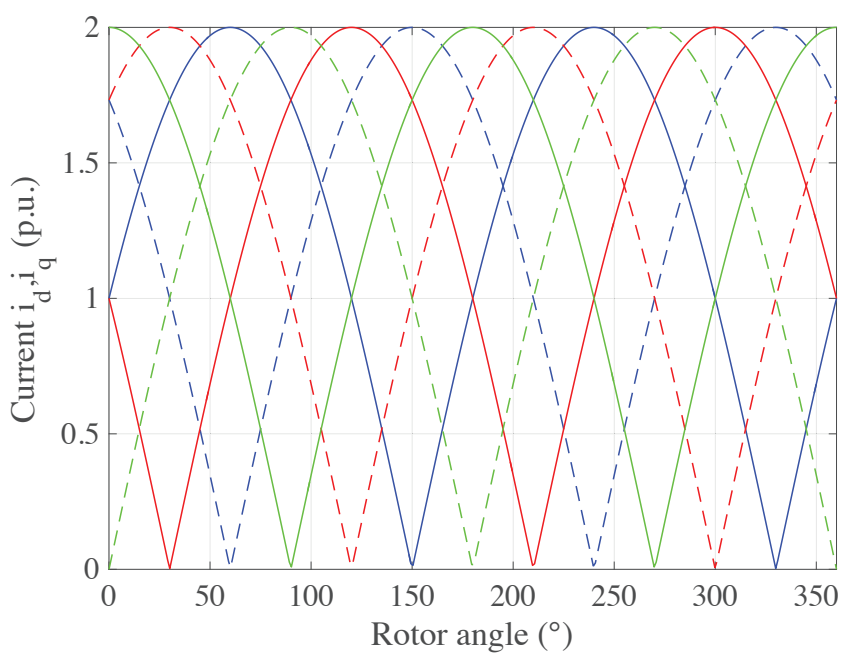

Fig. 7: Variation of the amplitude of the current $i_{d}$ and $i_{q}$ for different stator winding couplings. Blue curve: coupling I, red curve: coupling II and green curve: coupling III. Solid lines: d-axis current, dotted lines: q-axis current.

\section{A. DC-Decay I}

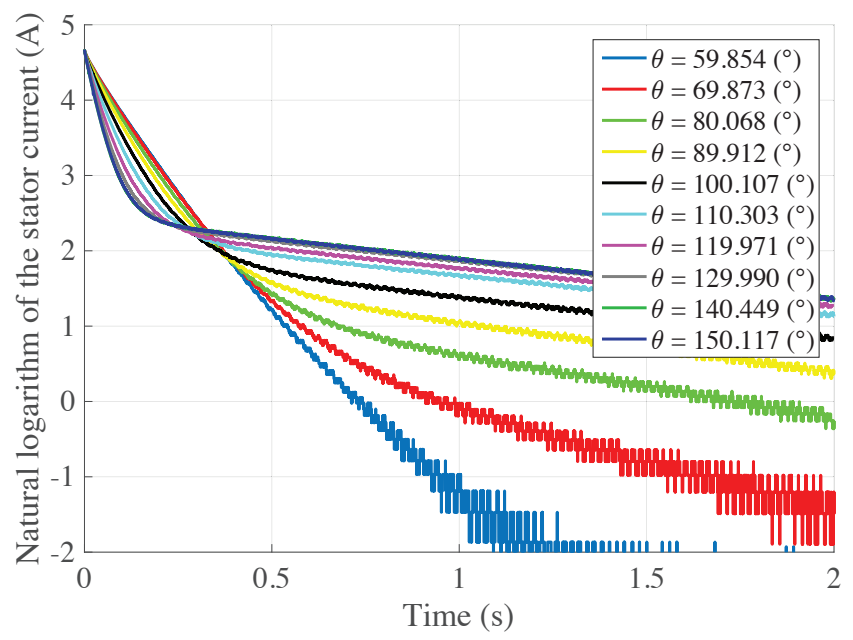

Fig. 8: Measured stator current for different rotor positions in the machine SM2, q-axis around $60^{\circ}$.

Fig. 8 shows the stator current for different rotor positions, which are in-line with the simulations. The poles are not clearly visible (straight lines) because SM2 has massive poles. The identification of the four poles is very difficult in practice, as the lumped element equivalent circuit is not suitable for the analytical description of the transient behaviour of massive poles. Plotting the natural logarithm of the current instead of the current lead to negative values in the y-axis. Fig. 9 presents the identified root locus for two poles of equation (2). As the measurements have been performed on a small power machine, the variation of the pole $\gamma$ is much higher than for SM1 while the pole $\beta$ behaves in a very similar way, as for SM1. The interpolated curve of the 

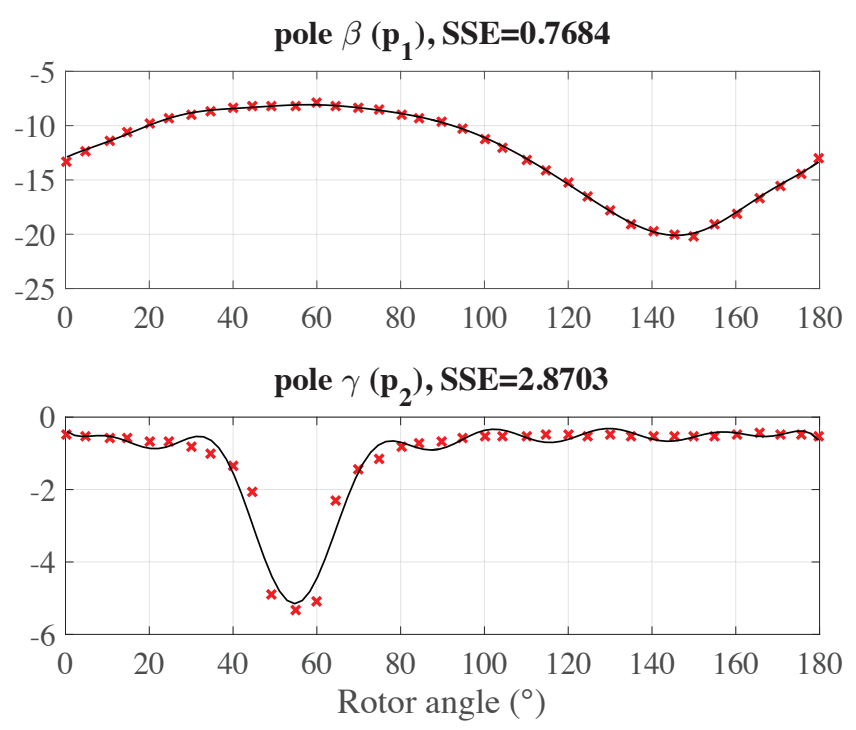

Fig. 9: Identified root locus in the machine SM2. Red crosses: identified poles, black line: interpolated curve (Fourierseries).

pole $\gamma$ does not fit well with the identified poles, illustrating the high and "step-like" variation of the root-locus instead of a relatively smooth and "sinus-like" angular variation. This could be due to a small air-gap or to the pole shoe shape.

\section{B. DC-Decay II}

The field current was set at about $65 \%$ of the no-load field current to get a good signal-to-noise ratio on the measured currents. $\theta_{e l}$ is the rotor angle obtained via the measurement method, $\theta_{\text {mes }}$ is the reference angle obtained using a position sensor and $\Delta \theta$ is the difference between these two values. If $\Delta \theta$ remains constant over the measurements then the novel method is correct. Table IV shows the obtained results.

TABLE IV: Determination of the rotor angle of the machine SM2.

\begin{tabular}{rrrl}
\hline$\theta_{e l}\left[{ }^{\circ}\right]$ & $\theta_{m e s}\left[{ }^{\circ}\right]$ & $\Delta \theta\left[^{\circ}\right]$ & $\mathrm{p}$-value [\%] \\
\hline 2.362 & 357.451 & 4.911 & $<1$ \\
24.010 & 18.984 & 5.025 & $<1$ \\
33.885 & 28.740 & 5.145 & $<1$ \\
70.982 & 66.094 & 4.799 & $<1$ \\
97.827 & 93.428 & 4.399 & $<1$ \\
199.128 & 194.063 & 5.066 & $<1$ \\
300.466 & 295.840 & 4.626 & $<1$ \\
\hline
\end{tabular}

The mean value of $\Delta \theta$ is $4.853^{\circ}$ and its standard deviation is $0.266^{\circ}$. The error on $\Delta \theta$ is distributed according a poisson distribution with rejection of the null hypothesis below $1 \%$ significance level, while each measurement of $\theta_{e l}$ is distributed according a normal distribution with rejection of the null hypothesis below $1 \%$ significance level ( $\mathrm{p}$-value in table IV). The variation of $\Delta \theta$ is $0.746^{\circ}$. All these indicators demonstrate the excellent precision of this novel method.

\section{DC-Decay III}

The poles of the decoupled transfer function are not identified due to the massive poles.. The demonstration is restricted to the separation of the current responses into the d- and q-axis for different rotor angles. Fig. 10 shows the time evolution of the phase currents.

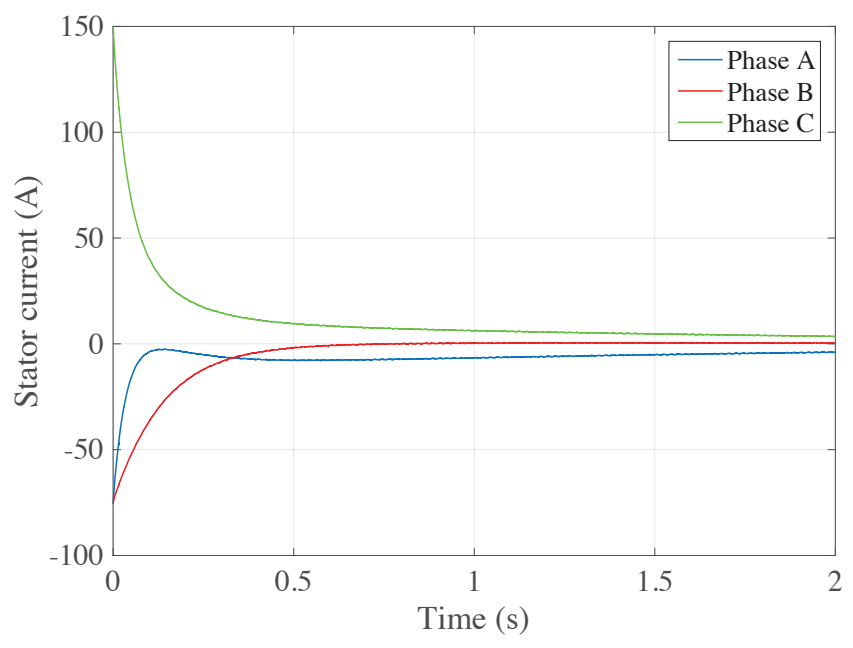

Fig. 10: Measured stator currents in the machine SM2.

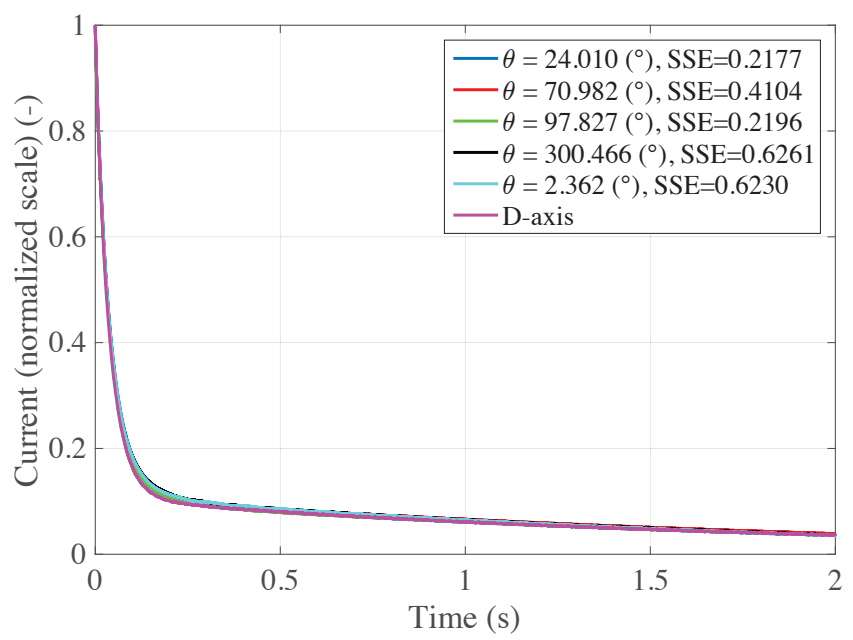

Fig. 11: Measured d-axis stator current in the machine SM2, SSE done on 20'000 samples with $i_{d}$ as reference.

Repeating this procedure for different rotor angles one obtains different current curves for both axes (see figures 11 and 12). The curves superpose themselves demonstrating the decomposition of the current curves into the 2 axes for an arbitrary rotor position. In the d-axis, the superposition is significantly worser than for the q-axis. Keeping in mind that the q-axis curves does not exhibit a similar discrepancy, the root cause of the discrepancy can certainly be attributed to the massive iron. The contribution of $x_{d}$ or the field winding seems to be differing with varying rotor angle. The mean value $(\bar{M})$ of the SSE for the d-axis is 0.4193 


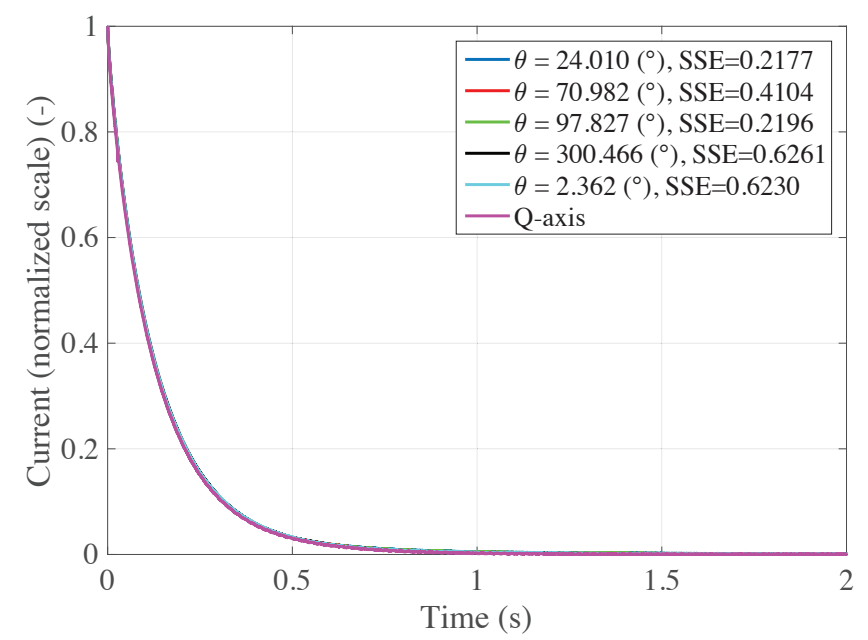

Fig. 12: Measured q-axis stator current in the machine SM2, SSE done on 20'000 samples with $i_{q}$ as reference.

and $\sigma=0.2030$, for the q-axis, $\bar{M}=0.1022$ and $\sigma=0.0343$. The cross-correlation between the SSE's for both axes is 0.9873 with a p-value of 0.0017 . The SSE's are strongly correlated demonstrating the accuracy of the measurement method, while the significant difference between their mean value is a demonstration of the discrepancies seen in Fig. 11.

\section{CONCLUSION}

The three new methods have been validated using simulations and measurement. The DC-Decay I method has a high angular resolution and gives an accurate root locus. This method has some drawbacks regarding the zero/pole simplification, which makes it a more "academical" option. There are significant discrepancies between the predicted root locus and the measured one. Therefore it could be considered if the transfer function described in equation (1) is a suitable transfer function for small power machines. The method DCDecay II shows small error on the rotor angle measurement.

The method DC-Decay III enables to catch the whole equivalent circuit with one single measurement, while up to now at least two measurements are required. This method should be compared to the sudden short-circuit at no-load to highlight the possible difference between the obtained values for the equivalent circuit in the d-axis and the influence of the saturation on the reactances and time constants through FE-simulations and measurements. Future work should apply the DC-Decay III method to a high power machine with laminated poles. Further, the measured curves presented in section VII should be identified using an analytical expression for the transfer function of massive poles.

\section{REFERENCES}

[1] I.M. Canay, Determination of model parameters of synchronous machine, IEEE Proc., vol. 130, pp. 86-94, Mar. 1983.

[2] I.M. Canay, Causes of Discrepancies on Calculation of Rotor Quantities and Exact Equivalent Diagrams of the Synchronous Machine, IEEE Trans. on Power Apparatus and Systems, vol. PAS-88, p.1114-1120, Jul. 1969.
[3] IEEE Standard 115, Test Procedures for Synchronous Machines, 1995.

[4] IEC 60034-4, Rotating electrical machines - Part 4: Methods for determining synchronous machine quantities from tests, 2008.

[5] E.S. Boje, J.C. Balda, R.G. Harley, R.C. Beck, Time-domain identification of synchronous machine parameters from simple standstill tests, IEEE Trans. Energy conversion, vol. 5, pp. 164-175, Mar. 1990.

[6] M.A. Arjona, M. Cisneros-Gonzalez and C. Hernandez, Development of a Synchronous-Generator Experimental Bench for Standstill TimeDomain Tests, Journal of Applied Research and Technology, vol. 9, pp. 117-128, 2011.

[7] F.S. Sellschopp and M.A. Arjona, DC decay test for estimating d-axes synchronous machine parameters: a two-transfer-function approach, IEE Proc.-Electr. Power Appl., Vol. 153, No. 1, January 2006.

[8] M. Vahedi, A. Hassania, H. Lotfian, Unique Solution For Dynamic Parameters Identification of a Synchronous Machine Using DC Decay Test, Journal of Electrical Engineering, Vol. 3, pp. 249-257, Edition 3, 2013.

[9] M. Cisneros-Gonzalez, C. Hernandez, R. Escarela-Perez and M. A. Arjona, Determination of Equivalent-circuit Parameters of a Synchronous Generator Based on the Standstill DC Decay Test and a Hybrid Optimization Method, Electric Power Components and Systems, 39:645?659, 2011.

[10] T. Kano, Y. Watanabe, T. Ara and T. Matsumura, Calculation of Equivalent Circuit Constants of Synchronous Machines Considering Field Transient Characteristics Using DC Decay Testing Method with Open and Shorted Field Windings, Electrical Engineering in Japan, Vol. 178, No. 2, 2012.

[11] P.J. Turner, A.B.J. Reece, D.C. Macdonald, The D.C. Decay Test for Determining Synchronous Machine Parameters: Measurement and Simulation, IEEE Transactionson Energy Conversion,Vol. 4, No. 4, December 1989.

[12] L. Majka, D. Szuster, Application of the stationary DC decay test to industrial turbogenerator model parameter estimation, PRZEGL'D ELEKTROTECHNICZNY, ISSN 0033-2097, R. 90 NR 4/2014.

[13] M. Hasni, O. Touhami, R. Ibtiouen, M. Fadel and S. Caux, Synchronous machine parameter identification by various excitation signals, Electr Eng (2008) 90:219:228.

[14] J.-J. Simond, I. Viorel, M. Tu Xuan, R. Wetter, L. Vicol, On the Identification of the Synchronous Machine Parameters Using Standstill DC Decay Test, ICEM, Chania, Crete Island, Greece, 2006.

[15] P. De Oliveira e Silva, Modal Analysis Applied to the Stability Study of Hydroelectric Systems with Modular Structures, Ph.D thesis n 6780, EPFL, Lausanne, Switzerland, 2015.

[16] F. Maurer, Determination des parametres de la machine synchrone par essais DC-Decay, M.S. thesis, Dep. elec., EPFL, Lausanne, Switzerland, 2009.

\section{BIOGRAPHIES}

Frederic Maurer received his master degree in 2009 from the Swiss Federal Institute of Technology in Lausanne (EPFL). At the time, he is pursuing a $\mathrm{PhD}$ in the field of "Electrical effect on AC windings of large DFIM". Since april 2009, he is working for Alstom (now GE) in different positions.

Mai Tu Xuan received his master degree in 1970 and his Ph.D. degree in 1977 from the EPFL. For many years he is senior researcher and lecturer at EPFL. His main fields of activities concern machine modeling, optimization and testing, parameter identification, measurement techniques and field calculations.

Jean-Jacques Simond received his master degree in 1967 and his Ph.D. degree in 1976 from the EPFL. Untill 1990 he has been working for BBC / ABB in the field of large electrical machines. Since 1990 he is full professor at the EPFL. He is also consultant for different international electrical machines manufacturers and utilities. 